\title{
A THEOREM ON RULED SURFACES
}

\author{
BY A. F. CARPENTER
}

On page 229 of volume 1 of Fubini-Čech, Geometria Proiettiva Differenziale, appears the following theorem.

If the two flecnode curves of a ruled surface are plane, then the complex curves and the harmonic curves are plane, and all of their planes belong to a pencil.

The authors furnish a proof of this, and they call it "an interesting theorem by Sullivan."

In a letter to the author of this note, Professor C. T. Sullivan says, with reference to the above, "The theorem you refer to is consequently not to be found in any of my publications." It appears probable that the Italian geometers have confused this theorem with one of a somewhat similar nature published by the author in $1915,{ }^{*}$ or perhaps with a somewhat more general theorem contained in a later paper by the author. $\dagger$

In this paper it is shown that the planes osculating the two branches of the flecnode curve and the planes osculating the two branches of the complex curve at the four points in which these curves cut a line element of their supporting ruled surface, will form a pencil, if and only if,

$$
D \equiv p_{21}^{2} \Delta_{1}-p_{12}^{2} \Delta_{2}=0,
$$

where

$$
\Delta_{1}=\stackrel{2}{p_{12}} q_{22}-p_{12}{ }_{12}^{\prime}+3 \stackrel{2}{q_{12}}, \Delta_{2} \equiv \stackrel{2}{p_{21}} q_{11}-p_{21} q_{21}^{\prime}+3 q_{21}^{2},
$$

$p_{i k}, q_{i k}$ being coefficients of the flecnode canonical form of the system of differential equations defining the surface. The theorem of 1915 is a special case of that of 1923 since

*Transactions of this Society, vol. 16 (1915), p. 520. This volume also contains a paper by Professor Sullivan.

$\nmid$ Tôhoku Mathematical Journal, vol. 23 (1923), p. 114. 
$\Delta_{1}=0, \Delta_{2}=0$ are necessary and sufficient conditions that the flecnode curves be plane.

It is the further purpose of this note to extend the theorem of the Tôhoku paper to include the harmonic curves.*

In the notation there employed the harmonic points are given by the factors of the covariant $p_{21} y^{2}+p_{12} z^{2}$. If $\eta=\left(p_{21}\right)^{1 / 2} y+i\left(p_{12}\right)^{1 / 2} z, \zeta=\left(p_{21}\right)^{1 / 2} y-i\left(p_{12}\right)^{1 / 2} z$, then the equations of the two planes osculating the curves $C_{\eta}, C_{\zeta}$, at points $P_{\eta}, P_{\zeta}$, are respectively.

$$
\begin{aligned}
p_{21}\left(p_{12}\right)^{1 / 2}\left(p_{12} p_{21}\left(p_{12} p_{21}\right)^{1 / 2}\right. & +i P)\left(i\left(p_{12}\right)^{1 / 2} x_{1}-\left(p_{21}\right)^{1 / 2} x_{2}\right) \\
& +f_{1}(i) x_{3}+f_{2}(i) x_{4}=0 \\
p_{21}\left(p_{12}\right)^{1 / 2}\left(p_{12} p_{21}\left(p_{12} p_{21}\right)^{1 / 2}\right. & -i P)\left(i\left(p_{12}\right)^{1 / 2} x_{1}+\left(p_{21}\right)^{1 / 2} x_{2}\right) \\
- & f_{1}(-i) x_{3}-f_{2}(-i) x_{4}=0
\end{aligned}
$$

where

$$
\begin{gathered}
f_{1}(i) \equiv D+2 p_{12} q_{21} P-p_{12}^{3} p_{21}^{3} \\
-i p_{12} p_{21}\left(p_{12} p_{21}\right)^{1 / 2}\left(2 p_{12} q_{21}+P\right), \\
\left(p_{12}\right)^{1 / 2} f_{2}(i) \equiv i\left(p_{21}\right)^{1 / 2}\left(D+2 p_{21} q_{12} P-p_{12}^{3} p_{21}^{3}\right) \\
+p_{12}^{2} p_{21}^{2}\left(p_{12}\right)^{1 / 2}\left(2 p_{21} q_{12}-P\right) \\
P=p_{12} q_{21}-p_{21} q_{12} .
\end{gathered}
$$

Two of the pencil of planes determined by (2), (3) are seen to be

(4) $\quad\left(\stackrel{3}{p_{12} p_{21}^{3}}+P^{2}\right)\left(p_{12} x_{2}+p_{12}^{2} x_{3}-2 q_{12} x_{4}\right)$

$$
\begin{aligned}
& -p_{12} D\left(p_{12}^{2} p_{21} x_{3}-P x_{4}\right)=0, \\
& \left(\stackrel{3}{p_{12} p_{21}^{3}}+P^{2}\right)\left(p_{21} x_{1}-2 q_{21} x_{3}+p_{21}^{2} x_{4}\right) \\
& -p_{21} D\left(P x_{3}-p_{12} p_{21}^{2} x_{4}\right)=0 .
\end{aligned}
$$

If, and only if, $D=0$, will (4), (5) reduce to

$$
\begin{aligned}
& p_{12} x_{2}+p_{12}^{2} x_{3}-2 q_{12} x_{4}=0, \\
& p_{21} x_{1}-2 q_{21} x_{3}+p_{21}^{2} x_{4}=0 .
\end{aligned}
$$

But (6) and (7) are the equations of the planes osculating

\footnotetext{
${ }^{*}$ These curves were called by Wilczynski the involute curves. See his Projective Differential Geometry, p. 208.
} 
the flecnode curves. Combining this result with the theorem of the Tôhoku paper we have the following theorem.

Theorem. The planes osculating the flecnode curve, the complex curve and the harmonic curve at the six points in which these curves cut a line element of their supporting ruled surface, will belong to a pencil, if, and only if,

$$
D \equiv \stackrel{2}{p_{21} \Delta_{1}}-\stackrel{2}{p_{12} \Delta_{2}}=0 \text {. }
$$

Since, when $\Delta_{1}=\Delta_{2}=0$, the flecnode curves are plane, the theorem of Fubini-Čech appears as a special case of the preceding theorem.

The University of Washington

\section{NETS OF CONICS IN THE GALOIS FIELDS OF ORDER $2^{n *}$}

\section{BY A. D. CAMPBELL}

We consider a net of conics in the Galois fields of order $2^{n}$ (1)

$$
\lambda C_{1}+\mu C_{2}+\nu C_{3}=0,
$$

where

$C_{i} \equiv a_{i} x^{2}+b_{i} y^{2}+c_{i} z^{2}+f_{i} y z+g_{i} z x+h_{i} x y, \quad(i=1,2,3)$, and where $C_{i}$ has the discriminant $\dagger$

$$
\Delta_{i} \equiv f_{i} g_{i} h_{i}+a_{i} f_{i}^{2}+b_{i} g_{i}^{2}+c_{i} h_{i}^{2} .
$$

Such a field is denoted for brevity by the symbol $G F\left(2^{n}\right)$. Along with (1) we consider the cubic curve in $\lambda, \mu, \nu$ that is obtained by equating to zero the discriminant of the general conic of (1) $\ddagger$

* Presented to the Society, December 31, 1926.

$\dagger$ See A. D. Campbell, Plane cubic curves in the Galois fields of order $2^{n}$, Annals of Mathematics, vol. 27 (1926), p. 395.

łCompare C. Jordan, Réduction d'un réseau de formes quadratiques, Journal des Mathématiques, (6), vol. 2 (1906), p. 412. 